\title{
Search for forced oscillations in binaries ${ }^{\star}$
}

\section{The eclipsing binary V436 Per revisited}

\author{
J. Janík ${ }^{1}$, P. Harmanec ${ }^{2,3}$, H. Lehmann ${ }^{4}$, S. Yang ${ }^{5}$, H. Božić ${ }^{6}$, H. Ak ${ }^{7}$, P. Hadrava ${ }^{3}$, P. Eenens ${ }^{8}$, D. Ruždjak ${ }^{6}$, \\ D. Sudar ${ }^{6}$, I. Hubeny ${ }^{9}$, and A. P. Linnell ${ }^{10}$ \\ 1 Institute of Theoretical Physics and Astrophysics, Faculty of Science, Masaryk University, Kotlářská 2, 61137 Brno, \\ Czech Republic \\ 2 Astronomical Institute of the Charles University, V Holešovičkách 2, 18000 Praha 8, Czech Republic \\ 3 Astronomical Institute, Academy of Sciences of the Czech Republic, 25165 Ondřejov, Czech Republic \\ e-mail: hec(had)@sunstel . asu.cas.cz \\ 4 Thüringer Landessternwarte Tautenburg, 07778 Tautenburg, Germany \\ e-mail: lehm@tls-tautenburg.de \\ 5 Dept. of Physics and Astronomy, University of Victoria, PO Box 3055, Victoria BC V8W 3P6, Canada \\ e-mail: yang@beluga.phys.uvic.ca \\ ${ }^{6}$ Hvar Observatory, Faculty of Geodesy, Zagreb University, Kačićeva 26, 10000 Zagreb, Croatia \\ e-mail: hbozic(rdomagoj, dsudar)@geodet.geof.hr \\ 7 Ankara University, Science Faculty, Astronomy and Space Science Dept., Tandoğan, Ankara 06100, Turkey \\ e-mail: ak@astro1.science.ankara.edu.tr \\ 8 Departamento de Astronomia, Universidad de Guanajuato, Apartado 144, 36000 Guanajuato, GTO, Mexico \\ e-mail: eenens@astro.ugto.mx \\ 9 National Optical Astronomy Observatories, PO Box 26732, 950 N. Cherry Ave., Tucson, AZ 85726-6732, USA \\ e-mail: hubeny@noao.edu \\ 10 Department of Physics and Astronomy, Michigan State University, E. Lansing, MI, 48824 and Visiting Scholar, Department \\ of Astronomy, University of Washington, Seattle, WA 98195, USA \\ e-mail: linnell@astro.washington.edu
}

Received 24 March 2003 / Accepted 27 May 2003

\begin{abstract}
An analysis of new spectroscopic and photoelectric $U B V$ observations, satisfactorily covering the whole orbital period of V436 Per, together with existing data allowed us to improve the knowledge of the basic physical characteristics of the binary and its components. In several aspects, our new results differ from the findings of Paper I of this series: in particular, we found that it is the star eclipsed in the secondary minimum which is slightly more massive and larger than the optical primary. We also conclude that the apsidal advance - if present at all - is much slower than that estimated in a previous study. The orbital period might be increasing by $0.28 \mathrm{~s}$ per year but also this finding is very uncertain and needs verification by future observations. It is encouraging to note that two completely independent sets of programs for light-curve solutions lead to identical results. A notable finding is that both binary components rotate with very similar - if not identical - rotational periods of 1.45 and 1.40 , much shorter than what would correspond to a 10.9 spin-orbit synchronization period at periastron. Rapid line-profile changes reported earlier could not be confirmed from new, dedicated series of high-resolution and S/N spectra.
\end{abstract}

Key words. stars: binaries: close - stars: binaries: eclipsing - stars: binaries: spectroscopic - stars: fundamental parameters stars: oscillations - stars: individual: V436 Per $\equiv 1$ Per

\section{Introduction}

This paper is a continuation of the series of SEFONO papers aimed at the search for forced oscillation in binaries (Harmanec et al. 1997; Paper I hereafter, Holmgren et al. 1997, 1999).

Send offprint requests to: J. Janík, e-mail: honza@physics . muni.cz

* This research is based on spectra from the Dominion Astrophysical Observatory (DAO), Ondřejov, and Thưringer Landessternwarte (TLS) Tautenburg.
V436 Per (1 Per, HR 533, HD 11241, BD+54³96; $V=$ $\left.5^{\mathrm{m}} .53, B-V=-0^{\mathrm{m}} \cdot 18, U-B=-0^{\mathrm{m}} .82\right)$ has been identified as an eclipsing binary by Kurtz (1977) who used it as a comparison star in his Strömgren $y$ observations of the Am star HR 540 but only French amateur observers succeeded in deriving its orbital period of $25^{\text {d }} 9$ - see Figer \& Maurin (1979). North \& Rufener (1981) noted that the amplitude of the RV curve was much lower than that expected for an eclipsing 
binary with a B-star primary. This problem was solved in Paper I: the first application of the KOREL disentangling technique to V436 Per led to the finding that the observed spectrum consist of heavily blended lines of two similar B2 stars. The correct RV curves have semiamplitudes of about $100 \mathrm{~km} \mathrm{~s}^{-1}$ and lead to normal masses for both stars. One longer series of electronic spectra used in Paper I also seemed to exhibit rapid line-profile variations.

The main goals of the present study are, therefore, to confirm or deny the presence of rapid line-profile variations of V436 Per reported in Paper I and to improve the knowledge of its basic physical characteristics, using new dedicated series of high-S/N spectroscopic and $U B V$ photometric observations.

\section{Observations and reductions}

\subsection{Spectroscopy}

Altogether, 199 new electronic spectra of V436 Per were obtained at three observatories:

- 2 in Ondřejov (2.0-m reflector, coudé, $17 \AA \mathrm{mm}^{-1}$ Reticon spectra, 6300-6700 ̊);

- 23 at the Dominion Astrophysical Observatory (DAO hereafter) (1.2-m reflector, coudé, $10 \AA \mathrm{Am}^{-1}$ CCD $4096 \mathrm{spec}-$ tra, covering the range 6100-6700 $\AA$ ); and

- 174 (consisting of whole-night series, see Table 10) in the TLS Tautenburg (2.0-m reflector, coudé, $3.2 \AA \mathrm{mm}^{-1}$ echelle spectra, 4700-7085 ̊).

Initial reductions of the spectra and their conversion into 1-D images were carried out by SY who used IRAF, for the DAO, by HL, using MIDAS software, for the Tautenburg spectra and by JJ using SPEFO software, developed by the late Dr. J. Horn - see Horn et al. (1996) and Škoda (1996). All subsequent reductions and velocity measurements were carried out by JJ in SPEFO. The wavelength calibrations were based on ThAr comparison spectra and the zero point of the wavelength scale was corrected individually through the measurements of selected atmospheric lines; see Horn et al. (1996) for details. Thanks to this last step, the spectra from all three instruments are safely on the same heliocentric wavelength scale for all practical purposes.

We combined the new spectra with the electronic spectra already used in Paper I. Additionally, we also used the published radial velocites, based on photographic spectra, and compiled already in Paper I (Harmanec et al. 1997). The journal of all RV data files is presented in Table 1.

The line spectrum of V436 Per is not very rich in the red spectral region. The only two strong lines are $\mathrm{H} \alpha$ and He I 6678. One can also note weak Si II 2 lines at 6347 and $6371 \AA$, Ne I 1 line at $6402 \AA$, and C II 2 lines at 6578 and $6583 \AA$, located in the red wing of the $\mathrm{H} \alpha$ line.

\subsection{Photometry}

The analyses of Paper I suffered from the lack of $U$ and $B$ observations covering the secondary eclipse of the binary. For that reason, 881 new $U B V$ observations were obtained
Table 1. Journal of RV observations.

\begin{tabular}{rcrc}
\hline \hline Source & $\begin{array}{c}\text { Epoch } \\
(\mathrm{JD}-2400000)\end{array}$ & $\begin{array}{r}\text { No. of } \\
\text { obs. }\end{array}$ & $\begin{array}{c}\text { Dispersion } \\
\left(\AA \mathrm{mm}^{-1}\right)\end{array}$ \\
\hline 1 & $19055.7-19409.7$ & 4 & $?$ \\
2 & $19662.7-20828.6$ & 59 & 40 \\
3 & 20016.8 & 1 & 30 \\
4 & $20369.9-21092.8$ & 8 & 33 \\
5 & $24042.9-30043.6$ & 4 & 30 \\
& $30732.6-30733.6$ & 2 & 51 \\
6 & $35794.6-35833.6$ & 16 & 34 \\
\hline 7 & $49327.3-50326.5$ & 16 & 17.2 \\
8 & $49744.6-51109.0$ & 47 & 10.0 \\
9 & $50056.9-50057.0$ & 6 & 10.0 \\
10 & $51245.2-51472.6$ & 174 & 3.2 \\
\hline
\end{tabular}

Details on sources of data and instruments used: 1 ...Abt (1970): Mt. Wilson 1.5-m reflector, prism spg.; 2 ...Beardsley (1969): Allegheny 0.79-m Keeler Memorial reflector, 1-prism Mellon spg.; 3 ...Frost et al. (1926): Yerkes 1.02-m refractor, Bruce 1-prism spg.; 4 ...Cannon (1918): Ottawa, 1-prism spg.; 5 ...Pearce \& Petrie (1951): Dominion Astrophysical Observatory 1.83-m reflector, 1-prism spg.; 6 ...Blaauw \& van Albada (1963): McDonald 2.08-m reflector, coudé grating spg.; 7 ...this paper: Ondřejov 2.0-m reflector, coudé grating spg. with a Reticon RL $1872 \mathrm{~F} / 30$ detector with $15 \mu$ m pixels; 8 ...this paper: DAO 1.22-m reflector, coudé grating spg. with a thick Loral $4096 \times 200 \mathrm{CCD}$ device with $15 \mu \mathrm{m}$ pixels; 9 ...this paper: DAO $1.83-\mathrm{m}$ reflector, Cassegrain grating spg. with a thick Loral $4096 \times$ $200 \mathrm{CCD}$ device with $15 \mu \mathrm{m}$ pixels; 10 ...this paper: Tautenburg 2.0$m$ reflector, coudé echelle spectrograph with a Tektronix $1024 \times 1024$ CCD with $24 \mu \mathrm{m}$ pixels.

at three observatories: at Hvar (0.65-m reflector, an uncooled EMI 6256 tube), San Pedro Mártir (SPM hereafter; 0.84-m reflector, Cuenta-pulsos photometer with a RCA 31034 tube cooled by dry ice) and the TÜBİTAK National Observatory (Turkey) (TNO hereafter; $0.40-\mathrm{m}$ reflector, SSP5A photometer). All these observations were obtained differentially, relative to 4 Per (HD 12303). A check star 2 Per (HD 11291) was observed as frequently as the variable. Observations from all three observatories were reduced with the help of the reduction program HEC22 (rel. 13 and 14) which uses non-linear formulæ for the transformation from the natural to the standard $U B V$ system; see Harmanec et al. (1994) and Harmanec and Horn (1998) for the description of observing and reduction procedures and for a detailed documentation of the reduction program. Additionally, we included Hipparcos $H_{\mathrm{p}}$ photometry published by Perryman et al. (1997), which we transformed into Johnson $V$ with the help of Harmanec's (1998) transformation formula.

We also used earlier $y$ and $U B V$ observations of V436 Per, already analyzed in Paper I, but with three exceptions: we omitted photometric observations by Güssow (1929) and by Percy (1982) which only cover the phases outside eclipses, and the less accurate $V$ observations by Böhme (1984). The Geneva photometry was transformed to Johnson $U B V$ using the transformation formulæ derived by Harmanec \& Božić (2001). The journal of all photoelectric observations on which this study is based is presented in Table 2 . 
Table 2. Journal of photoelectric observations.

\begin{tabular}{lcrllll}
\hline \hline Source & $\begin{array}{c}\text { Epoch } \\
\text { (JD-2400000) }\end{array}$ & $\begin{array}{r}\text { No. of } \\
\text { obs. }\end{array}$ & Passband(s) & Comparison & Check \\
\hline Kurtz (1977) & $42706.7-42744.7$ & 91 & $y$ & HR 540 & HR 502 \\
North et al. (1981) & $37190.5-42076.3$ & 159 & $U B$ & $\begin{array}{l}\text { all sky } \\
\text { all sky }\end{array}$ & - \\
& $37190.5-44626.4$ & 281 & $V$ & HR 540 & 4 Per \\
Poretti (1982) & $44919.3-45000.4$ & 102 & $V$ & HR 540 & 4 Per \\
Poretti (1984) & $45621.4-45741.3$ & 419 & $V$ & HR 540 & 4 Per \\
and Paper I & $46348.3-46348.5$ & 48 & $V$ & all sky & - \\
\hline Hipparcos & $47871.9-49040.6$ & 108 & $V$ & 4 Per & 2 Per \\
\hline Hvar & $50086.2-52561.5$ & 196 & $U B$ & 4 Per & 2 Per \\
Tübitak & $50086.2-52561.5$ & 195 & $V$ & 4 Per & 2 Per \\
San Pedro Mártir & $51051.6-51059.5$ & 92 & $U B V$ & 4 Per & 2 Per \\
\hline
\end{tabular}

Table 3. $U B V$ data for the comparison and check star used.

\begin{tabular}{|c|c|c|c|c|}
\hline \multicolumn{5}{|l|}{4 Per (comp.) } \\
\hline$V$ & $B-V$ & $U-B$ & Station & Remark \\
\hline $5^{\mathrm{m}} \cdot 012 \pm 0^{\mathrm{m}} \cdot 011$ & $-0 \mathrm{~m} .078$ & $-0^{\mathrm{m}} \cdot 309$ & Hvar & all-sky \\
\hline \multicolumn{5}{|l|}{ HR 540 (comp.) } \\
\hline $6^{\mathrm{m}} \cdot 450 \pm 0^{\mathrm{m}} .006$ & $0^{\mathrm{m}} \cdot 183$ & $00^{\mathrm{m}} \cdot 108$ & Hvar & all-sky \\
\hline \multicolumn{5}{|l|}{2 Per (check) } \\
\hline $5^{\mathrm{m}} \cdot 716 \pm 0^{\mathrm{m}} \cdot 009$ & $-0^{\mathrm{m}} .070$ & $-0^{\mathrm{m}} \cdot 277$ & Hvar & all-sky \\
\hline $5^{\mathrm{m}} \cdot 715 \pm 0^{\mathrm{m}} .009$ & $-0^{\mathrm{m}} .070$ & $-0^{\mathrm{m}} \cdot 278$ & Hvar & dif. \\
\hline $5^{\mathrm{m}} .711 \pm 0^{\mathrm{m}} \cdot 006$ & $-0^{\mathrm{m}} .071$ & $-0^{\mathrm{m}} \cdot 268$ & SPM & dif. \\
\hline $5^{\mathrm{m}} .713 \pm 0^{\mathrm{m}} \cdot 008$ & $-0^{\mathrm{m}} .071$ & $-0^{\mathrm{m}} .269$ & TNO & dif. \\
\hline
\end{tabular}

To bring all data into a comparable system of standard $U B V$ magnitudes, we added the all-sky magnitudes of 4 Per and HR 540, based on numerous Hvar observations and listed in Table 3, to the respective magnitude differences V436 Per comparison and 2 Per - comparison. Mean differential $U B V$ values of the check star 2 Per from all three observatories are also listed in Table 3 to illustrate the accuracy of our homogenization.

For convenience for future investigators, we publish all the homogenized individual photoelectric observations in Table $4^{1}$.

\section{Solution of the light curve}

\subsection{FOTEL}

To obtain a new solution of the light curve, we used the program FOTEL (Hadrava 1990) and all $U, B, V$ and $y$ observations listed in Table 2. Some convenient features of FOTEL are that it allows calculation of individual zero points for individ-

\footnotetext{
1 Table 4 is only available in electronic form at CDS via anonymous ftp to cdsarc.u-strasbg.fr $(130.79 .128 .5)$ or via http://cdsweb.u-strasbg.fr/cgi-bin/qcat?J/A+A/408/611
}

ual data sets and also a simultaneous solution for observations in several different bandpasses.

One of the conclusions of Paper I was that V436 Per may undergo a slow apsidal motion with a period of $12300 \pm 1900$ years. Additional photometric observations of the narrow eclipses that we secured allow us to carry out further checks on that finding. A simultaneous FOTEL solution using all $U B V y$ observations and all KOREL RVs yields a significantly smaller value of the periastron advance, which corresponds to a period of $51800 \pm 9400$ years. According to the covariance matrix which is a part of FOTEL solution, this value is somewhat correlated with a possible secular increase of the orbital period estimated by FOTEL as $(0.26 \pm 0.06) \mathrm{s} \mathrm{yr}^{-1}$. Regarding also other strong correlations among several elements (for instance eccentricity, epoch of periastron passage and longitude of periastron are all correlated with correlation coefficients close to 0.99 ) one can conclude that the actual uncertainties of the above values are larger than what their rms errors, estimated from the covariance matrix, seem to imply. This means that both the apsidal advance and the period change are only marginally significant and tests on their reality by future observations of binary eclipses are required.

To enable such tests, we derived a FOTEL solution that neglects the very slow apsidal advance but allows for a period change as one of the elements. This solution indeed gives a small but formally significant increase of period with time, $\dot{P}=0.284 \pm 0.064 \mathrm{~s} \mathrm{yr}^{-1}$ which leads to the following quadratic ephemeris for the epochs of primary minima:

$$
\begin{array}{r}
T_{\text {prim.min. }}=\text { HJD } 2443562.861+25.935918 \cdot E \\
+1.167364 \times 10^{-7} \cdot E^{2} .
\end{array}
$$

We note, however, that the rms of this solution is practically identical to that for the solution without a period change.

Considering the above discussion and the fact that the suspected period change is almost negligible over the time interval covered by our data, we decided to carry out further analyses assuming a constant period and no apsidal advance.

We then proceeded in two steps. First, we calculated a solution in which all data were treated with equal weight. In the second step we assigned each data set with a weight estimated 


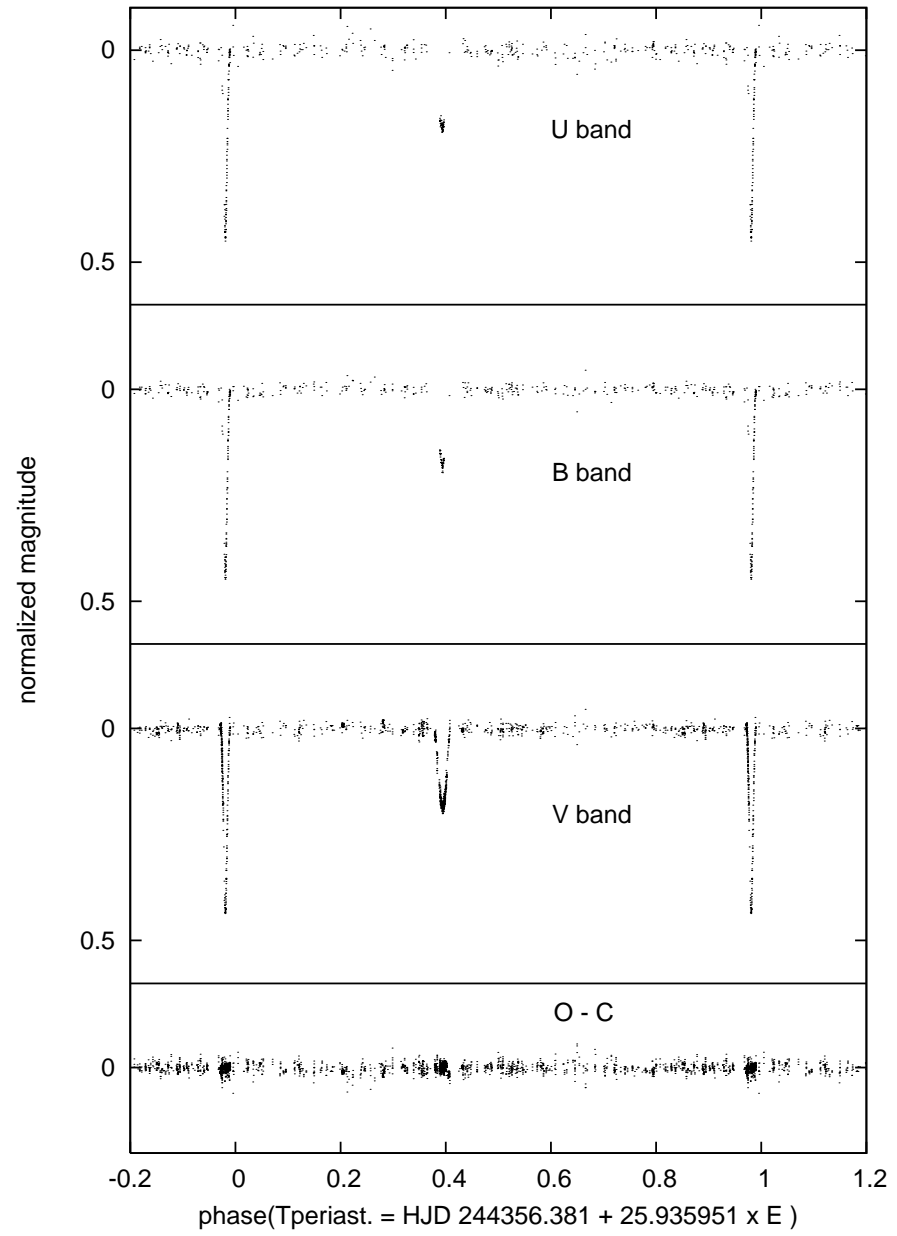

Fig. 1. Light curves of V436 Per, based on all observations in $U, B$ and $V$ passbands and the $\mathrm{O}-\mathrm{C}$ deviations from the theoretical curves based on solution 2 of Table 5 .

from the first solution. Weights were taken to be inversely proportional to the square of the rms errors. We fixed the linear limb-darkening coefficients at values of 0.390 in $U, 0.395$ in $B$ and 0.340 in $V$, adopted from Claret (2000). We let the program converge automatically, to machine accuracy, to achieve the solution after several hundreds of iterations. Both unweighted and weighted solutions are presented in detail in Table 5 .

The $U, B$ and $V+y$ light curves are shown in Fig. 1 together with the $\mathrm{O}-\mathrm{C}$ deviations of all $U B V y$ observations from the final theoretical light curve.

\subsection{BINSYN}

As a separate check on the $V$ solution, we used the BINSYN suite of programs (Linnell \& Hubeny 1996) to do an independent simulation of the system. The BINSYN suite uses a different simulation algorithm from FOTEL. The BINSYN suite adopted the system parameters listed in Tables 7 and 9. In the first simulation we adopted the linear limb darkening coefficient 0.340 used in the FOTEL solution. The plot of residuals was similar to that of Fig. 1, and the calculated rms $V$ residual of 1 observation was 0.0100 , i.e. practically the same as for solution 1 of Table 5. In a second simulation,
Table 5. Simultaneous solutions of the $U B V$ light curves assuming a constant orbital period. All epochs are in HJD-2 400000.

\begin{tabular}{|c|c|c|}
\hline Element & $\begin{array}{l}\text { Solution } 1 \\
\text { unweighted }\end{array}$ & $\begin{array}{l}\text { Solution } 2 \\
\text { weighted }\end{array}$ \\
\hline$P(\mathrm{~d})$ & 25.935953 & 25.935951 \\
\hline $\mathrm{rms}$ & 0.000003 & 0.000003 \\
\hline$T_{\text {periastr. }}$ & 43563.384 & 43563.381 \\
\hline rms & 0.013 & 0.009 \\
\hline$T_{\text {prim.min. }}$ & 43562.861 & 43562.861 \\
\hline$T_{\text {sec.min. }}$ & 43573.574 & 43573.576 \\
\hline$e$ & $0.4012 \pm 0.0037$ & $0.4023 \pm 0.0025$ \\
\hline$\omega\left({ }^{\circ}\right)$ & $108.39 \pm 0.23$ & $108.31 \pm 0.16$ \\
\hline$i\left(^{\circ}\right)$ & $88.073 \pm 0.002$ & $88.049 \pm 0.002$ \\
\hline$r_{1}$ & $0.03454 \pm 0.0012$ & $0.03495 \pm 0.0010$ \\
\hline$r_{2}$ & $0.04714 \pm 0.0007$ & $0.04721 \pm 0.0007$ \\
\hline$V_{\text {McDonald }}$ & $5.5236 \pm 0.0008$ & $5.5235 \pm 0.0008$ \\
\hline rms & 0.0077 & 0.0076 \\
\hline$V_{\text {Geneva }}$ & $5.5322 \pm 0.0007$ & $5.5320 \pm 0.0007$ \\
\hline rms & 0.0115 & 0.0117 \\
\hline$V_{\text {Merate }}$ & $5.5179 \pm 0.0004$ & $5.5182 \pm 0.0004$ \\
\hline $\mathrm{rms}$ & 0.0102 & 0.0103 \\
\hline$V_{\mathrm{Hvar}}$ & $5.5276 \pm 0.0005$ & $5.5279 \pm 0.0005$ \\
\hline $\mathrm{rms}$ & 0.0072 & 0.0068 \\
\hline$V_{\mathrm{TNO}}$ & $5.5257 \pm 0.0007$ & $5.5252 \pm 0.0007$ \\
\hline $\mathrm{rms}$ & 0.0066 & 0.0065 \\
\hline$V_{\text {SanPedroMartir }}$ & $5.5252 \pm 0.0019$ & $5.5252 \pm 0.0019$ \\
\hline rms & 0.0047 & 0.0047 \\
\hline$V_{\text {Hipparcos }}$ & $5.5299 \pm 0.0006$ & $5.5298 \pm 0.0006$ \\
\hline $\mathrm{rms}$ & 0.0061 & 0.0061 \\
\hline$B_{\text {Geneva }}$ & $5.3545 \pm 0.0010$ & $5.3545 \pm 0.0010$ \\
\hline $\mathrm{rms}$ & 0.0128 & 0.0128 \\
\hline$B_{\text {Hvar }}$ & $5.3498 \pm 0.0005$ & $5.3499 \pm 0.0005$ \\
\hline $\mathrm{rms}$ & 0.0071 & 0.0069 \\
\hline$B_{\mathrm{TNO}}$ & $5.3456 \pm 0.0008$ & $5.3454 \pm 0.0008$ \\
\hline $\mathrm{rms}$ & 0.0074 & 0.0073 \\
\hline$B_{\text {SanPedroMartir }}$ & $5.3461 \pm 0.0018$ & $5.3461 \pm 0.0018$ \\
\hline rms & 0.0043 & 0.0043 \\
\hline$U_{\text {Geneva }}$ & $4.5209 \pm 0.0013$ & $4.5209 \pm 0.0013$ \\
\hline $\mathrm{rms}$ & 0.0161 & 0.0161 \\
\hline$U_{\mathrm{Hvar}}$ & $4.5083 \pm 0.0008$ & $4.5084 \pm 0.0008$ \\
\hline $\mathrm{rms}$ & 0.0113 & 0.0113 \\
\hline$U_{\mathrm{TNO}}$ & $4.5248 \pm 0.0009$ & $4.5246 \pm 0.0009$ \\
\hline $\mathrm{rms}$ & 0.0084 & 0.0084 \\
\hline$U_{\text {SanPedroMartir }}$ & $4.5286 \pm 0.0027$ & $4.5286 \pm 0.0027$ \\
\hline $\mathrm{rms}$ & 0.0067 & 0.0067 \\
\hline mean rms & 0.0101 & 0.0088 \\
\hline No. of obs. & 2248 & 2248 \\
\hline
\end{tabular}

The "mean rms" is the mean rms error of one observation of unit weight for the solution; all errors quoted with individual elements are rms errors of these elements calculated from the cross-correlation matrix.

we used non-linear second-order limb darkening coefficients (Wade \& Rucinski 1985). This simulation produced a calculated rms $V$ residual of 0.0099 .

Because of the large eccentricity of the system, it is conceivable that the mutual irradiation varies sufficiently with orbital position to affect the light curves. We used BINSYN to 
Table 6. The final light-curve solution of Table 5 is compared with the orbital solutions from KOREL. All epochs are in HJD-2 400000.

\begin{tabular}{llll}
\hline \hline element & photometry & $\begin{array}{l}\text { KOREL } \\
\text { He I 6678 }\end{array}$ & $\begin{array}{l}\text { KOREL } \\
\mathrm{H} \alpha\end{array}$ \\
\hline$P(\mathrm{~d})$ & $25.935951 \pm 0.000003$ & fixed & fixed \\
$T_{0}$ & $43563.381 \pm 0.009$ & 43563.367 & 43563.362 \\
$e$ & $0.4023 \pm 0.0025$ & 0.383 & 0.377 \\
$\omega(\mathrm{deg})$ & $108.31 \pm 0.16$ & 108.8 & 108.6 \\
$K_{1}\left(\mathrm{~km} \mathrm{~s}^{-1}\right)$ & - & 98.0 & 97.5 \\
$K_{2}\left(\mathrm{~km} \mathrm{~s}^{-1}\right)$ & - & 93.1 & 90.0 \\
\hline
\end{tabular}

calculate the temperature change at the substellar point, on each component, between mid-primary minimum and midsecondary minimum. For component 1 , at mid-primary minimum, the substellar point $T_{\text {eff }}$ is $21532.7 \mathrm{~K}$; for component 2 the value is $22017.2 \mathrm{~K}$. At mid-secondary minimum, the $T_{\text {eff }}$ of the substellar point on component 1 is $21505.0 \mathrm{~K}$ and for component 2, 21999.7 K. We defer further study of this effect to a later differential correction solution with BINSYN.

In the rest of this paper, we shall adopt the value of the orbital period based on solution 2 of Table 5 .

\section{Spectrum analysis}

We analyzed two different spectral regions by a method of spectral disentangling (Hadrava 1995, 1997), using a new version of the program KOREL which allows one to use up to 300 spectra in the solution. Two different spectral regions were analyzed separately to check the consistency of the results.

The first region (6538-6594 A, see Fig. 2 lower part) contains an $\mathrm{H} \alpha$ and $\mathrm{C}$ II doublet and numerous telluric lines. For numerical reasons, it is necessary to use a larger step in RV for spectral segments covering broad lines like $\mathrm{H} \alpha^{2}$. In particular, we used a step of $5.0 \mathrm{~km} \mathrm{~s}^{-1}$ per bin and derived a solution in which the decomposition of telluric lines was also included.

The second region (6670-6687 $\AA$, see Fig. 2 upper part) contains only the line of He I 6678 and could be analyzed with a finer step of $1.5 \mathrm{~km} \mathrm{~s}^{-1}$ per bin. This region is free of telluric lines and was therefore decomposed into only two binary components.

During these solutions, the orbital period was fixed at a value known with high accuracy from the weighted solution of the combined $U B V y$ light curve, given in Table 5. KOREL solutions for both spectral regions are compared with the final FOTEL light-curve solution in Table 6 and the RV curves for the He I line are shown in Fig. 3. These solutions are in very good agreement with each other and with the photometric solution.

\footnotetext{
${ }^{2}$ As explained in the original paper on KOREL, this program works with data representation in the logarithmic scale in wavelength. Its present version has a limited field to store the digitized line profiles. It is, therefore, important to use a step in wavelength that fully covers the studied spectral line, or a blend of lines, but does not reduce the original spectral resolution more than necessary.
}

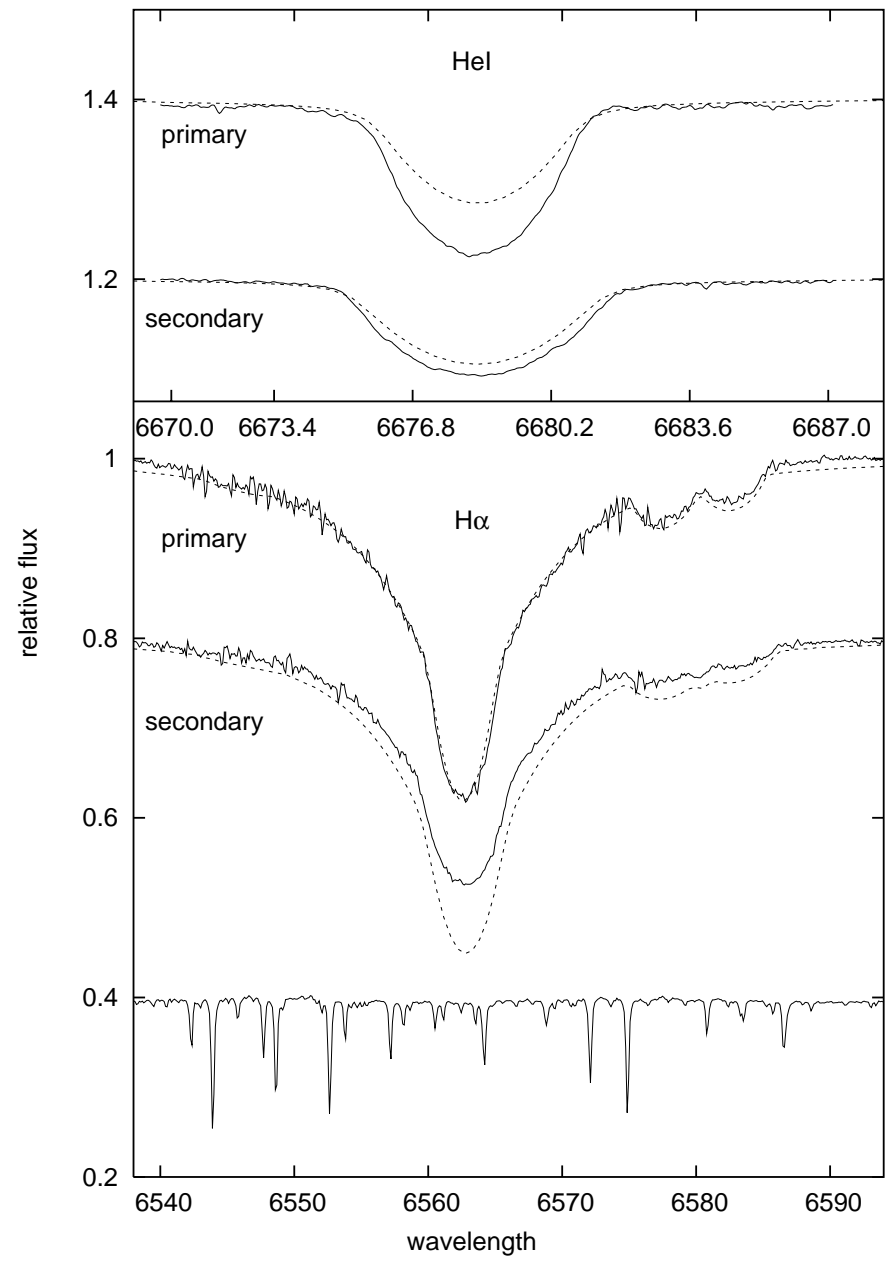

Fig. 2. The decomposition of $\mathrm{H} \alpha$ and He I 6678 and their comparison with best fit theoretical line profiles (primary: $T_{\text {eff }}=21500 \mathrm{~K}$, $\log g=4.25$, secondary: $T_{\text {eff }}=22000 \mathrm{~K}$ and $\left.\log g=4.14\right)$. Projected rotational velocities of theoretical line profiles are 115 and $140 \mathrm{~km} \mathrm{~s}^{-1}$ for the primary and secondary, respectively. The curve at the bottom is the mean spectrum of telluric lines.

\section{Basic physical characteristics of the binary}

\subsection{Masses, radii and radiative characteristics}

To obtain the final values of the masses, radii and other basic physical elements of the binary, we adopted RVs of the He I 6678 line from the KOREL solution, considering that this line could be decomposed with a finer step in RV per pixel and gives, therefore, a more accurate result. Then we used FOTEL to calculate the final set of elements, based on a simultaneous solution of the light and RV curves. Relative weights of photometry and spectroscopy were chosen in such a way that photometry and spectroscopy accounted for half of the sum of $(\mathrm{O}-\mathrm{C})^{2}$ each. The results of this combined solution are given in Table 7 (orbital and photometric elements) and in Table 9 (basic physical elements).

One can see that the result of Paper I was altered: it is the smaller and less massive star that is eclipsed in the primary 


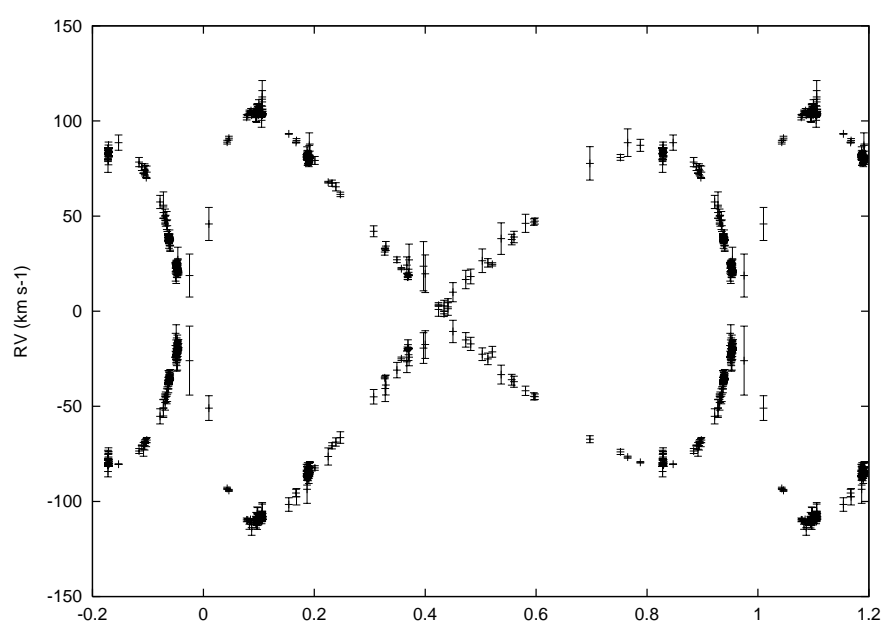

Fig. 3. RV curves of both binary components based on a KOREL solution for the He I 6678 line. Larger errors of a few data points are related to phases of binary eclipses.

Table 7. Final set of the orbital and photometric elements derived from the combined RV and light-curve solution with FOTEL.

\begin{tabular}{|c|c|}
\hline$P(\mathrm{~d})$ & 25.935951 fixed \\
\hline$T_{\text {periastr. }}(\mathrm{HJD}-2400000)$ & $43563.466 \pm 0.005$ \\
\hline$T_{\text {prim.min. }}(\mathrm{HJD}-2400000)$ & 43562.861 \\
\hline$T_{\text {sec.min. }}(\mathrm{HJD}-2400000)$ & 43573.577 \\
\hline$e$ & $0.3768 \pm 0.0014$ \\
\hline$\omega\left(^{\circ}\right)$ & $109.83 \pm 0.10$ \\
\hline$K_{1}\left(\mathrm{~km} \mathrm{~s}^{-1}\right)$ & $97.4 \pm 0.1$ \\
\hline$K_{2}\left(\mathrm{~km} \mathrm{~s}^{-1}\right)$ & $91.2 \pm 0.1$ \\
\hline$M_{1} \sin ^{3} i\left(M_{\odot}\right)$ & 6.94 \\
\hline$M_{2} \sin ^{3} i\left(M_{\odot}\right)$ & 7.41 \\
\hline$A \sin i\left(R_{\odot}\right)$ & 89.54 \\
\hline$i\left(^{\circ}\right)$ & $88.048 \pm 0.002$ \\
\hline$r_{1}$ & $0.03676 \pm 0.00024$ \\
\hline$r_{2}$ & $0.04308 \pm 0.00050$ \\
\hline$L_{1}(U)$ & 0.409 \\
\hline$L_{1}(B)$ & 0.410 \\
\hline$L_{1}(V)$ & 0.412 \\
\hline $\operatorname{rms}\left(\mathrm{km} \mathrm{s}^{-1}\right)$ & 2.92 \\
\hline rms (mag) & 0.0089 \\
\hline
\end{tabular}

minimum ${ }^{3}$. We think that the reason for high sensitivity of the result to the quality and quantity of photometric data stems also from the unique geometry of the binary: the orbit is highly eccentric and one of the eclipses occurs quite close to the periastron passage. This may explain the rather large observed difference in the depths of both eclipses.

\footnotetext{
${ }^{3}$ We should mention that FOTEL can also converge to another solution resulting in a larger radius for the less massive star. However, since this solution gives a larger rms error and leads also to a larger difference in the calculated reddening for the two stars, we prefer the solution given in Table 7.
}

Table 8. Observed $U B V$ magnitudes of V436 Per and dereddened magnitudes and colours of both binary components; see the text for details.

\begin{tabular}{lccl}
\hline \hline Quantity & Observed & Primary & Secondary \\
\hline$V$ & $5^{\mathrm{m}} \cdot 5264$ & $6^{\mathrm{m}} \cdot 3254$ & $5^{\mathrm{m}} \cdot 9564$ \\
$B$ & $5^{\mathrm{m}} \cdot 3471$ & $6^{\mathrm{m}} \cdot 0840$ & $5^{\mathrm{m}} \cdot 7392$ \\
$U$ & $4^{\mathrm{m}} \cdot 5238$ & $5^{\mathrm{m}} \cdot 2390$ & $4^{\mathrm{m}} \cdot 8718$ \\
$B-V$ & $-0^{\mathrm{m}} \cdot 1793$ & $-0^{\mathrm{m}} \cdot 2414$ & $-0^{\mathrm{m}} \cdot 2172$ \\
$U-B$ & $-0^{\mathrm{m}} \cdot 8233$ & $-0^{\mathrm{m}} \cdot 8450$ & $-0^{\mathrm{m}} \cdot 8674$ \\
$E(B-V)$ & - & $0^{\mathrm{m}} \cdot 0294$ & $0^{\mathrm{m}} \cdot 0667$ \\
$E(U-B)$ & - & $0^{\mathrm{m}} \cdot 0211$ & $0^{\mathrm{m}} \cdot 0478$ \\
\hline
\end{tabular}

Table 9. Basic physical elements of V436 Per.

\begin{tabular}{lll}
\hline \hline Element & Primary & Secondary \\
\hline$M / M_{\odot}{ }^{*}$ & 6.95 & 7.42 \\
$R / R_{\odot}{ }^{*}$ & 3.29 & 3.86 \\
$\log g[\mathrm{CGS}]$ & 4.25 & 4.14 \\
$T_{\text {eff }}(\mathrm{K})$ & 21500 & 22000 \\
$M_{\text {bol }}(\mathrm{mag})$ & -3.60 & -4.05 \\
$M_{\mathrm{V}}(\mathrm{mag})$ & -1.37 & -1.77 \\
$v_{\text {obs. }} \sin i\left(\mathrm{~km} \mathrm{~s}^{-1}\right)^{* *}$ & 115 & 140 \\
$P_{\text {rot. }}(\mathrm{d})$ & 1.45 & 1.40 \\
\hline
\end{tabular}

* Based on combined RV and light-curve FOTEL solution.

** Based on rotationally broadened NLTE line profiles.

\subsection{Effective temperature, reddening, rotational velocities and distance}

We adopted the mean $V, B$ and $U$ magnitudes of the binary outside minima as a weighted mean of these values derived by FOTEL for all data sets transformed to the standard $U B V$ system (cf. Table 5). They are given in the column "Observed" of Table 8. Using the luminosity ratios derived by FOTEL for each passband, we derived the $U B V$ magnitudes of both binary components and applied a standard dereddening. Individually derived values of the reddening are given in columns "Primary" and "Secondary" for star 1 and 2, respectively. The dereddened values of $U B V$ magnitudes of both components, given in the same columns, were derived using the mean values of the reddening derived for the primary and secondary, $E(B-V)=$ 0.048 and $E(U-B)=0.035$.

To obtain a preliminary estimate of the effective temperatures of both stars, we used the correlation between $(B-$ $V)$ and $\log T_{\text {eff }}$ derived by Buser \& Kurucz (1978) and by Popper (1980). This led to $T_{\text {eff } 1}=(21500 \pm 250) \mathrm{K}$ and $T_{\text {eff } 2}=$ $(22000 \pm 250) \mathrm{K}$ for the primary and secondary, respectively. These values correspond well to the often - quoted spectral class B2.

To check on the estimates of the effective temperatures, we compared the line profiles decomposed by KOREL with the new NLTE line-blanketed model atmosphere computed 
with TLUSTY (Hubeny 1988; Hubeny \& Lanz 1995), and the SYNSPEC program (Hubeny et al. 1994) to generate model atmosphere fluxes. A plane parallel geometry was assumed in these calculations, as well as hydrostatic and radiative equilibria.

From a comparison with the line profiles decomposed by KOREL we also derived the $v \sin i$ values for both stars. The observed (disentangled) and synthetic line profiles are compared in Fig. 2.

A NLTE model atmosphere spectrum corresponding to the parameters of the primary star $\left(T_{\text {eff }}=21500 \mathrm{~K}, \log g=4.25\right)$ was convolved with a rotational line profile having $v \sin i=$ $115 \mathrm{~km} \mathrm{~s}^{-1}$ for the secondary $\left(T_{\text {eff }}=22000 \mathrm{~K}, \log g=4.14\right)$ $v \sin i=140 \mathrm{~km} \mathrm{~s}^{-1}$. The agreement is excellent for the $\mathrm{H} \alpha$ profile of the primary and satisfactory for the He I 6678 profile of the secondary. The comparison is much less satisfactory for the $\mathrm{H} \alpha$ profile of the secondary and the He I 6678 profile of the primary.

We carried out various comparisons with NLTE line profiles derived for a broader range of effective temperatures from $18000 \mathrm{~K}$ to $24000 \mathrm{~K}$ but the result was not altered. We were unable to obtain a really satisfactory fit for both stellar lines from both components simultaneously. Note that the effective temperature and - to some extent - also the relative luminosities (their errors are of the order of 10 per cent) are the only parameters we could vary since both the gravity acceleration and luminosity ratios of the two compoments are tightly fixed by our new RV and light-curve solutions. We, therefore, leave this problem open for the moment, until new photometry in both minima and spectra over a broader range of wavelenghts are available.

Adopting the above effective temperatures and dereddened $V$ magnitudes of both stars, we were able to estimate also the distance to the binary, $d=(340 \pm 15) \mathrm{pc}$. This agrees very well with the direct distance determination, based on the parallax measured by the Hipparcos satellite (Perryman et al. 1997: $d=$ $(315 \pm 66) \mathrm{pc})$.

\section{Rapid line-profile changes}

Thanks to dedicated series of TLS spectra (5 nights, see Table 10), we could carry out a decisive test for the presence of rapid line-profile changes. To this end, we allowed KOREL to subtract the disentangled line profiles of both binary components (in $\mathrm{H} \alpha$ and $\mathrm{He}$ I 6678), properly shifted in $\mathrm{RV}$, from the individual spectra, and output the residual spectra in the rest frame of the primary, and secondary, respectively. For neither of these two possibilities did we detect any significant lineprofile variations.

To check on the presence of line-profile variations, we also used the temporal variance spectrum technique (TVS hereafter), devised by Fullerton et al. (1996). We calculated the self-TVS for each long series of spectra listed in Table 10, again with a completely negative result.

These exercises are illustrated by Figs. 5 and 4. On the left side of Fig. 5, there is part of the residual spectra from KOREL in the rest frame of the primary and the TVS of all these residual spectra. In the middle panel, there is part of the residual

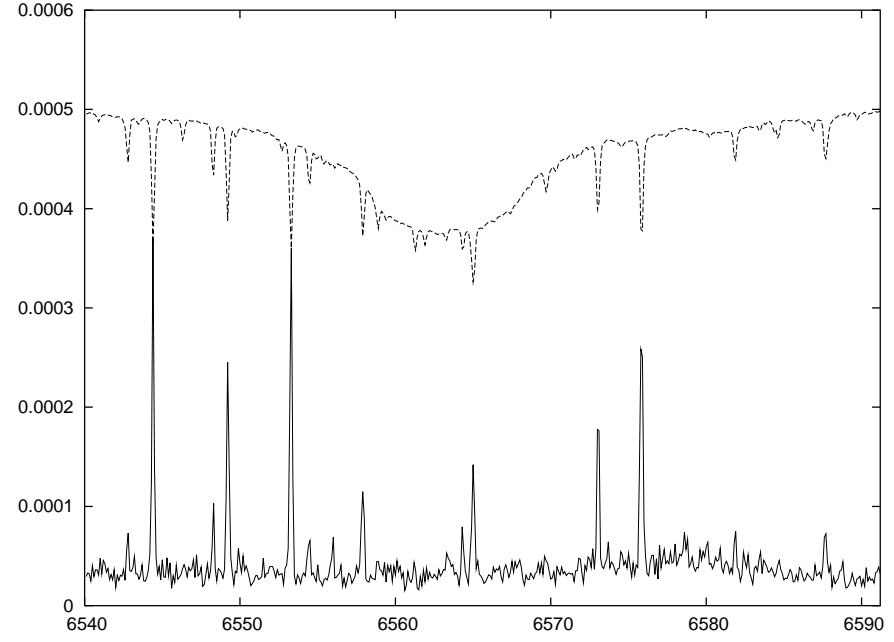

Fig. 4. Bottom: the temporal variance spectrum of $\mathrm{H} \alpha$ computed for the series of TLS spectra taken between HJD 2451450.3511 and HJD 2451450.6635. Top: the mean $\mathrm{H} \alpha$ line profile with the neighbouring telluric lines for the same series. Only telluric lines stand out in the temporal variance spectrum, their changes being caused by the rotation of the Earth and its revolution around the Sun.

Table 10. Journal of TLS spectra.

\begin{tabular}{ll}
\hline \hline Epoch (HJD-2 400 000) & No. of obs. \\
\hline $51245.2405-51245.3851$ & 42 \\
$51443.4225-51443.4785$ & 17 \\
$51446.2506-51446.3414$ & 27 \\
$51450.3511-51450.6635$ & 53 \\
$51472.5393-51472.6586$ & 35 \\
\hline
\end{tabular}

spectra in the rest frame of the secondary compontent and the TVS of all spectra again. Neither in the residual spectra nor in the temporal variance spectra did we find any significant changes. On the right side of the figure, there is part of the $o b$ served spectra and the temporal variance spectrum calculated for all observed spectra of V436 Per. Here, one can see a bump in the core of the $\mathrm{H} \alpha$ line. This is, however, caused only by the orbital motion of the binary.

In Fig. 4, the observed mean $\mathrm{H} \alpha$ profile is compared with the TVS for the longest (fourth) night series. Only small shifts of telluric lines caused by the Earth's rotation and revolution around the Sun are detected but no trace of line-profile variations of the stellar profile can be seen ${ }^{4}$.

We therefore conclude that the detection of line-profile changes, reported in Paper I, was spurious, caused by some accidental noise in the observed spectra.

\section{Discussion}

A new analysis of 243 electronic spectra and 2248 photoelectric observations of V436 Per allowed us to derive very

\footnotetext{
4 We tested the TVS technique on a night series of spectrograms of a well-known line-profile variable $\varepsilon$ Per. The line-profile variations were detected very convincingly in this case.
} 

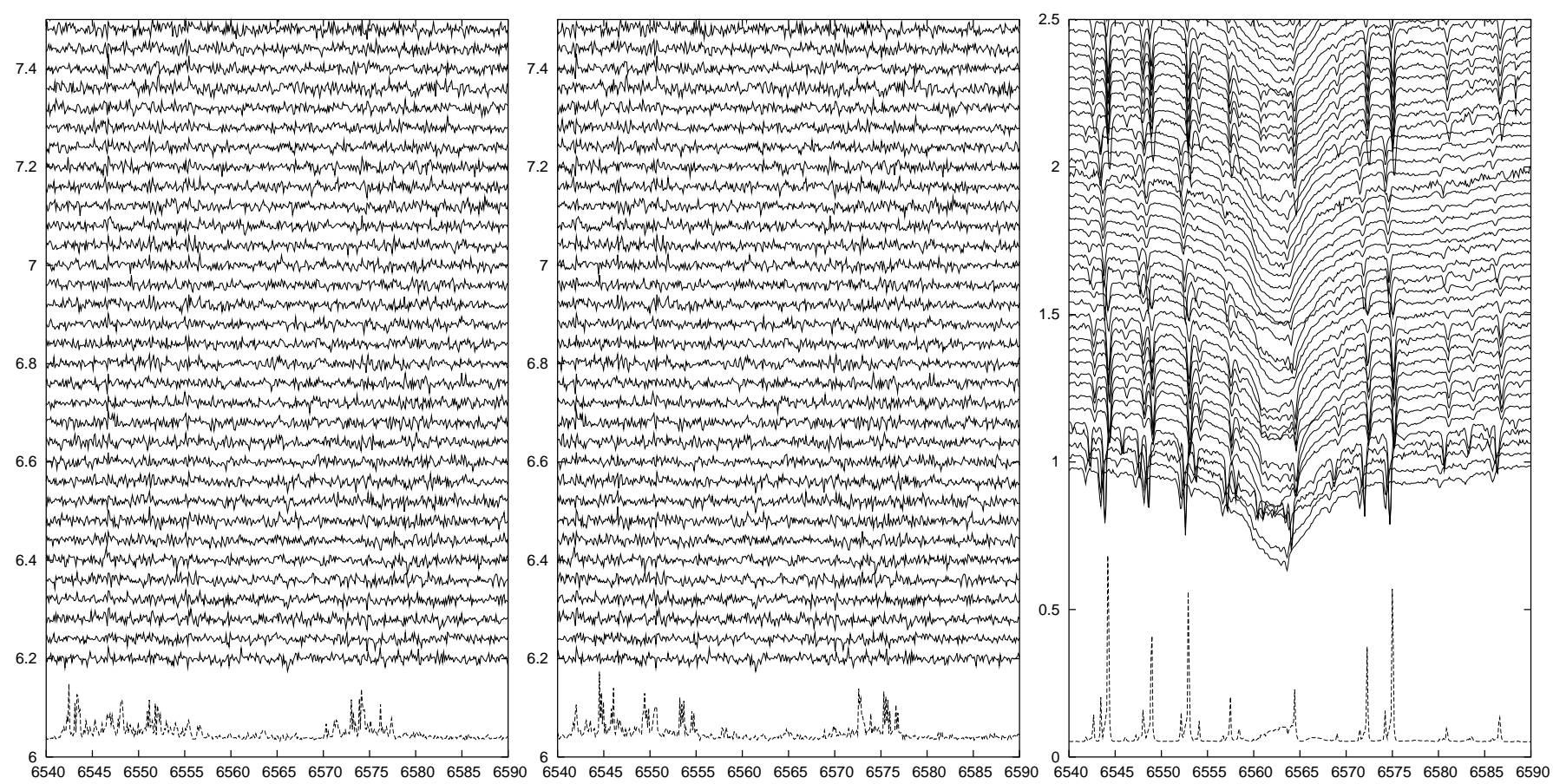

Fig. 5. Left: a part of residual spectra in the rest frame of the primary and the TVS of all these residual spectra. Middle: a part of residual spectra in the rest frame of the secondary and the TVS of all these residual spectra. Right: a part of observed spectra of V436 Per and the TVS of all of them. Telluric lines stand out in the TVS and there is also a bump in the core of $\mathrm{H} \alpha$. This, however, is solely due to the RV changes related to the orbital motion.

accurate physical characteristics of this binary. In contrast to Paper I, we found that the photometric primary is the smaller and less massive component of the binary. We also found that there is neither a measurable apsidal motion in the system nor evidence of rapid line-profile changes.

In spite of this, V436 Per still seems to be an astrophysically interesting system, found in a rather unusual dynamical state. Using the elements derived here, one can calculate the rotational periods of the primary and secondary, 1.45 and 1.40 , respectively. The synchronization at periastron would require rotational periods of both stars to be 10.9 (cf., e.g., Harmanec 1988). We thus have a binary system with two similar, but distinctly different binary components, which both rotate much faster than what synchronization at periastron would require and have different $v \sin i$ but very similar if not identical rotational periods.

A disturbing problem which remains unsolved after this new study is the obvious discrepancy between the theoretical fully-blanketed NLTE line profiles of $\mathrm{H} \alpha$ for the secondary, and for the He I 6678 line profile of the primary. The available electronic spectra are insufficient for a deeper discussion as we only have one Balmer line at our disposal. We therefore postpone the investigation of this problem for future study.

Acknowledgements. We acknowledge the suggestions by an anonymous referee which helped to improve the presentation of this paper. This study is partly based on the observational data secured in the course of the Czech-Mexican KONTAKT/CONACyT collaborative project E130.734. In the early stages, it was carried out as a part of the research supported by the grants 205/96/0162, 205/02/0445 and A3003805 of the Grant Agency of the Czech Republic. Its finishing was made possible through the support via research plans J13/98: 113200004 of MŠMT, and AV 0Z1 003909 and via project K2043105 of AV ČR. APL and IH would like to thank the Mobility Fund, Charles University, Prague, for the financial support during their stay in Prague and Ondřejov, and to all colleagues at the Astronomy Department of the Charles University for their hospitality.

\section{References}

Abt, H. A. 1970, ApJS, 19, 387

Beardsley, W. R. 1969, Publ. Allegheny Obs., 8, No. 7

Blaauw, A., \& van Albada, T. S. 1963, ApJ, 137, 791

Böhme, D. 1984, Inf. Bull. Var. Stars, No. 2507

Buser, R., \& Kurucz, R. L. 1978, A\&A, 70, 555

Cannon, J. B. 1918, Publ. Dominion Obs. Ottawa, 4, 253

Claret, A. 2000, A\&A, 363, 1081

Figer, A., \& Maurin, L. 1979, GEOS Circular on Eclipsing Binaries EB2, Paris, January 12, 1979

Frost, E. B., Barrett, S. B., \& Struve, O. 1926, ApJ, 64, 1

Fullerton, A. W., Gies, D. R., \& Bolton, C. T. 1996, ApJ, 103, 475

Güssow, M. 1929, Astron. Nachr., 237, 321

Hadrava, P. 1990, Contr. Astron. Obs. Skalnaté Pleso, 20, 23

Hadrava, P. 1995, A\&AS, 114, 393

Hadrava, P. 1997, A\&AS, 122, 581

Harmanec, P. 1988, Bull. Astron. Inst. Czechosl., 39, 329

Harmanec, P. 1998, A\&A, 335, 173

Harmanec, P., \& Božić, H. 2001, A\&A, 369, 1140

Harmanec, P., \& Horn, J. 1998, J. Astron. Data, 4, CD-ROM file 5

Harmanec, P., Horn, J., \& Juza, K. 1994, A\&AS, 104, 121

Harmanec, P., Hadrava, P., Yang, S., et al. 1997, A\&A, 319, 867 
Holmgren, D., Hadrava, P., Harmanec, P., Koubský, P., \& Kubát, J. 1997, A\&A, 322, 565

Holmgren, D., Hadrava, P., Harmanec, P., et al. 1999, A\&A, 345, 855

Horn, J., Kubát, J., Harmanec, P., et al. 1996, A\&A, 309, 521

Hubeny, I. 1988, Comp. Phys. Commun., 52, 103

Hubeny, I., \& Lanz, T. 1995, ApJ, 439, 875

Hubeny, I., Lanz, T., Jeffery, C. S. 1994, in Newsletter on Analysis of Astronomical Spectra, No. 20, ed. C. S. Jeffery (St. Andrews University), 30

Kurtz, D. W. 1977, PASP, 89, 939

Linnell, A. P., Hubeny, I. 1996, ApJ, 471, 958

North, P., Rufener, F., Figer, A., \& Maurin, L. 1981, Inf. Bull. Var. Stars, No. 2036
Pearce, J. A., \& Petrie, R. M. 1951, Publ. Dom. Astrophys. Obs., 8, 409

Percy, J. R. 1982, Inf. Bull. Var. Stars, No. 2085

Perryman, M. A. C., Høg, E., Kovalevsky, J., Lindegren, L., \& Turon, C. 1997, ESA SP-1200: The Hipparcos and Tycho Catalogues

Poretti, E. 1982, Inf. Bull. Var. Stars, No. 2239

Poretti, E. 1984, Inf. Bull. Var. Stars, No. 2529

Popper, D. M. 1980, ARA\&A, 18, 115

Škoda, P., 1996, in Astronomical Data Analysis Software and Systems V, ed. G. H. Jacoby, \& J. Barnes, ASP Conf. Ser., 101, 187

Wade, R. A., \& Rucinski, S. M. 1985, A\&AS, 60, 471 